\title{
The impact of SUZAKU on the knowledge of the physics of CVs
}

\section{Takayuki Hayashi*}

NASA, Goddard Space Flight Center, Code 662, Greenbelt, MD20771, USA

E-mail: takayuki.havashienasa.gov

\begin{abstract}
Suzaku satellite observed dozens of WD binaries and gave us various findings and knowledge. In this paper, four topics are picked up. (1) From a nova outburst of V2491 Cyg, a very hard non-thermal component with $\Gamma=0.1 \pm 0.2$ was found. Mass accretion seemed to be rekindled 30 days after the nova and its X-ray spectrum is similar to that of intermediate polars. (2) In a nova outburst of a symbiotic Mira star V407 Cyg, a non-equilibrium ionization (NEI) plasma emission was found, which shows asymmetry in the environment encountered by the nova ejecta. The coincidental $\mathrm{GeV} \gamma$-ray decline/X-ray rise can be explained by the stalling of the shock front because of the ejecta running into the red giant photosphere. (3) X-ray spectra of five symbiotic stars (CD-28 3719, Hen 3-1591, Hen 3-461, EG And and 4 Dra) have hot thermal plasma of $T_{\max }$ $\gtrsim 3 \mathrm{keV}$. To reproduce thetemperature, X-ray energy source should be ascribed to release of the gravitational potential in boundary layers. (4) X-ray spectrum of $\gamma$ Cas can be reproduced by a single-temperature thermal plasma model of $15 \mathrm{keV}$. X-ray dips of order of $10^{4} \mathrm{~s}$ can be explained by partial covering absorption. A WD binary is more likely for $\gamma$ Cas identity because of its spectrum without power-law component and consistency of the covering fractions of absorbers and dip duration.
\end{abstract}

The Golden Age of Cataclysmic Variables and Related Objects IV 11-16 September, 2017

Palermo, Italy

\footnotetext{
* Speaker.
} 


\section{Introduction}

White dwarfs (WDs) often emit X-rays with several energy sources, making binary systems. Cataclysmic variables (CVs) are WD binaries whose secondary star is a late-type main-sequence star [4]]. CVs whose WD has a weak magnetic field $(B<0.1 \mathrm{MG})$ are called non-magnetic CVs. In these systems, accreting gas forms an accretion disk extending close to the WD surface. The inner disk and the WD is so different in velocity and the accreting gas is heated up $\sim 10^{5-8} \mathrm{~K}$ by friction in the area between the disk and the WD. This area called a boundary layer in which the hot gas emits X-rays or EUV by thermal bremsstrahlung and line emission.

Magnetic CVs (mCVs) are the WD binaries whose WD magnetic field is so strong $(B>$ $0.1 \mathrm{MG}$ ) that it can catch the accreting gas and the accreting gas falls onto the WD along the magnetic field. The channeled accreting gas is heated up to $\sim 10^{8} \mathrm{~K}$ and highly ionized via a strong shock near the WD surface [8, [2]. The ionized gas emits X-ray and gets cold, approaching the WD surface, which called an accretion column. When the magnetic field is moderate $(0.1<B<10 \mathrm{MG})$, the X-ray binaries are called intermediate polars (IPs) [26]. In these systems, an accretion disk is partly formed and the accreting gas is caught around the Alfvén radius. Polars hold a WD of the strong magnetic field $(10<B<230 \mathrm{MG})$ which catches the accreting gas directly from the secondary and, therefore, the WD spin period synchronize with that of the revolution [ [ $\rightarrow$ ].

Some of the CVs having the disk show us dwarf nova outburst, which are called dwarf novae (DNe). This outburst is due to thermal-viscous instability in their disks [25]. In DNe, accreting matter is accumulating in their disk during quiescence. When amount of the accumulated gas reaches a threshold, the matter in the disk is ionized and its viscosity increases. At that moment, the accreting rate from the disk to the WD drastically increases and the brightness is enhanced with 2-5 mag lasting 2-20 days [40]. The outburst is quasi-periodic and its intervals is 10 days to tens of years.

Symbiotic stars (SSs) are binary systems of a red giant and a compact star which is mostly a WD. 45 symbiotic stars of the 220 have beed detected with X-ray [B]. X-ray spectrum of the symbiotic stars is some combination of four types [ㄷ]]. Three of them are thermal emission originated by the quasi-stable nuclear burning on the WD surface $(\alpha)$, the collision of wind with $T \lesssim 1 \mathrm{keV}$ $(\beta)$ and the release of the gravitational potential in the boundary layer like the non-magnetic CVs $(\delta)$. The other is power-law-type $(\gamma)$ of non-thermal origin, which is shown in symbiotic stars with a neutron star as the compact star [48]].

Nova explosions are a thermonuclear runaway event in accreted material on the WD surface [可]. This event is triggered by that the accreted mass reaches a critical mass and brightness of the progenitor is increased by $\sim 10 \mathrm{mag}$. Classical novae $(\mathrm{CNe})$ are the WD binaries in which the nova explosion was observed. In general, the novae explosion are expected to periodically occur on timescales of 10 to $10^{5}$ years. The timescale is determined by the WD mass and the accretion rate. More massive white dwarfs have higher surface gravities and higher pressures. As a result, the critical mass is reduced. The classical novae where the nova explosion was observed more than once are referred to as recurrent novae (RNe).

Suzaku X-ray astronomical satellite [ए]] had instruments favorable for the WD binary observations. Suzaku equipped X-ray CCDs coupled with X-ray telescopes, which have moderate energy resolution, large effective area and low background noise. The X-ray spectra of the WD binaries of- 
ten show prominent iron $\mathrm{K}$ emission lines of neutral to highly ionized plasma which give us plasma temperature, dynamics of the accreting matter and, geometry of the plasma and the WD. A high sensitivity hard X-ray $(>10 \mathrm{keV})$ detector is also mounted on Suzaku. It is useful for estimation of the maximum plasma temperature and intensity of scattered X-rays.

In $\S \rrbracket$, Suzaku satellite and its instruments are introduced. Several results with Suzaku are taken up in [3. Finally, those results are summarized in brief.

\section{Suzaku satellite}

Suzaku satellite is the fifth Japanese X-ray astronomical satellite and collaboration mission between Japan and the United States. It was launched at the Uchinoura Space Center of the Japan Aerospace Exploration Agency on July 10, 2005. The weight and the length of its spacecraft were 1.7 tons and $6.5 \mathrm{~m}$, respectively. Suzaku orbited around the Earth with the period of 96 minutes at $570 \mathrm{~km}$ altitude with the inclination angle of 32 degree and its pointing accuracy was about 0.2 arcmin. Suzaku had four X-ray CCDs called the X-ray Imaging Spectrometer (XIS) which is installed at foci of dedicated X-Ray Telescopes (XRTs) for each of them. The Hard X-ray Detector (HXD) onboard Suzaku actualize high sensitivity observation in hard X-ray above $10 \mathrm{keV}$. Suzaku completed its scientific operation in June 2015, however, high quality archival data has been accumulated.

Four modules of the XIS are designated as XIS0-3 [ए2]. Of them, XIS1 adopts a backilluminated (BI) CCD while the others employ front-illuminated (FI) CCDs. The FI and BI CCDs cover energy ranges of $0.4-10 \mathrm{keV}$ and $0.2-10 \mathrm{keV}$, respectively, and their energy resolutions are $\sim 130-250 \mathrm{eV}$ and $\sim 150-300 \mathrm{eV}$, respectively. Energy scale accuracy is typically $\leq 10 \mathrm{eV}$ at the $\mathrm{Mn}-\mathrm{K} \alpha$ line (5.895 keV). After the middle of 2006, the spaced-row charge injection technique was routinely operated which drastically improved the energy resolution [20, [38]. XIS2 and part of XIS0 have been unusable from November 2006 and June 2009, respectively, because of anomalies.

The XRTs adopt Wolter-I type grazing-incident reflective optics consisting of tightly nested, thin-foil conical mirror shells. The angular resolution ranges from 1.8 to 2.3 arcmin in half-power diameter. The effective area is $440 \mathrm{~cm}^{2}$ at $1.5 \mathrm{keV}$ and $250 \mathrm{~cm}^{2}$ at $8 \mathrm{keV}$ per module.

HXD is a non-imaging and collimated detector [33], [1]], which is composed of two types of detectors. One is the PIN detector which adopts $2 \mathrm{~mm}$-thick silicon PIN diodes and is sensitive in $10-70 \mathrm{keV}$. The other is the GSO/BGO phoswitch counter which is sensitive in 40-600 keV. The energy resolution is $3.0 \mathrm{keV}$ (FWHM) to the PIN detector and $7.6 / \sqrt{E} \%$ (FWHM) to the GSO detector, where $E$ is energy in $\mathrm{MeV}$. Since in general no significant flux was detected with the GSO in the WD binaries observation.

\section{Suzaku results}

Suzaku observed dozens of the WD binaries: 15 DNe [BG], 7 polars, 23 (or 24) IPs [4⿴囗⿰丿㇄口], 6 nova outbursts, 7 symbiotic stars [24] and, $3 \gamma$ Cas analogues. I note that WD binaries are candidates for identity of $\gamma$ Cas analogues. 4 topics are picked up and introduced in the following subsections. 


\subsection{V2491 Cygni}

V2491 Cygni is a CN and was discovered on 2008 April $10.728 \mathrm{UT}$ at $(l, b)=\left(67^{\circ} .22874,4^{\circ} .35315\right)$ [2]1, [28]. The evolution of the nova was extremely fast and its brightness was declined by 2 mag in 4.6 days $\left(t_{2} \sim 4.6 \mathrm{~d}\right)$ [37]. Its distance was roughly estimated at $10.5 \mathrm{kpc}$ with an empirical relation between the maximum magnitude and the declining rate [5]. The extremely fast evolution shows the WD mass is on the higher end of the WDs [Q].

Figure $\mathbb{W}$ shows a $\mathrm{K} \alpha$ emission line of He-like iron and a very flat continuum up to $70 \mathrm{keV}$ in the X-ray spectrum observed by Suzaku 9 days after the start of the nova (reproduction from [B5]]). The presence of the He-like iron and absence of the $\mathrm{H}$-like iron indicate that the thermal plasma temperature is of $\lesssim 10 \mathrm{keV}$, which, however, can not create the flat continuum. Moreover, bremsstrahlung of electron temperature of even $100 \mathrm{keV}$ can not reproduce the flat continuum (blue line in figureW). Instead, the spectrum is reproduced by a combination of $2.9_{-2.6}^{+4.3} \mathrm{keV}$ thermal plasma and power-law emission with a photon index of $0.1 \pm 0.2$ attenuated by a strong absorption of $N_{H}=1.4_{-0.4}^{+0.9} \times 10^{23} \mathrm{~cm}^{-2}$. This is the first clear detection of non-thermal X-ray signature from novae, which shows novae accelerate high-energy particles. However, mechanisms of acceleration and radiation are unclear.

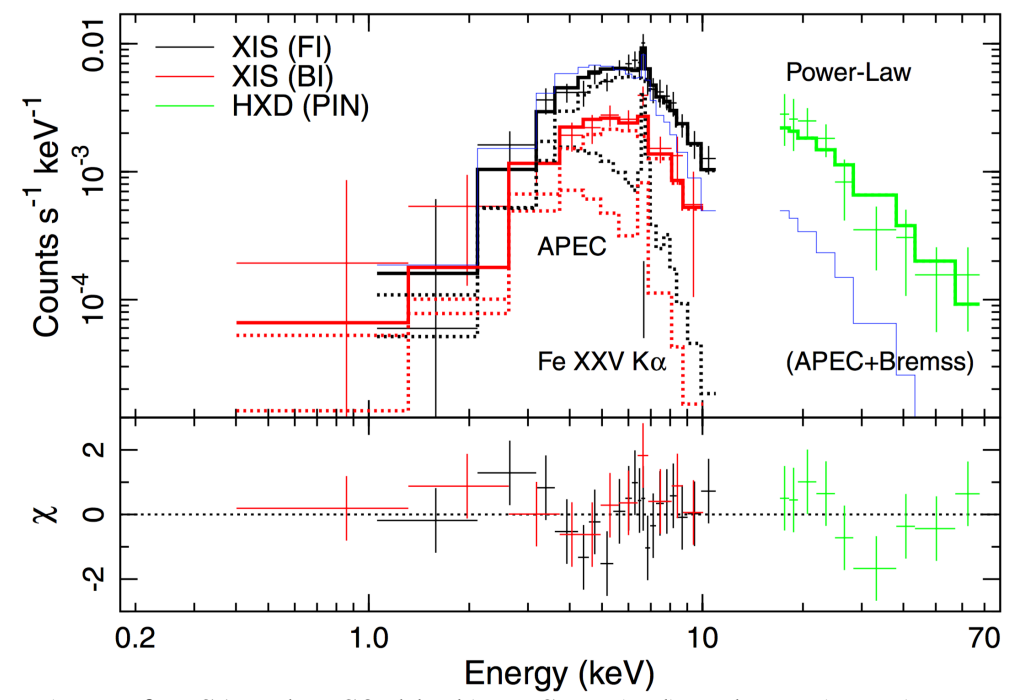

Figure 1: XIS-FI (sum of XIS1 and XIS3; black), XIS-BI (red) and PIN (green) spectra of V2492 Cyg on 9 days after the arise of the nova. The best-fit model (thermal plasma + power-law) is shown with thick lines (solid for total and dashed for each component). The alternative unsuccessful model (APEC +100 $\mathrm{keV}$ bremsstrahlung) is shown with thin blue lines. The lower panel shows the residual from the best fit. (Reproduction from fig.4 of [35]])

Iron $\mathrm{K} \alpha$ lines evolve as well as the brightness in the nova outburst. The soft X-ray flux (0.3$4.0 \mathrm{keV}$ ) was negligible just after the start of the outburst but grew up and reach the peak 40 days after the start. By contrast, the hard X-ray flux is relatively stable. Figure $\square$ shows the evolution of the iron $\mathrm{K} \alpha$ lines of its neutral, He-like and H-like atom observed by Suzaku, XMM-Newton and Swift satellites (reproduction from [B] ]). The $\mathrm{K} \alpha$ line of neutral iron was significant only after the peak. The origin of X-ray before the peak is commonly interpreted as internal shocks (i.e. ejecta with different velocities collide with each other). On the other hand, the origin of 
$\mathrm{X}$-ray after the peak was proposed to be due to rekindled accretion by [36]. In fact, the X-ray features of the later phase is similar to those of IPs at several aspects: (1) The $\mathrm{K} \alpha$ emission lines of neutral, He- and H-like are clearly seen. (2) The continuum emission is very flat (T $\sim 10 \mathrm{keV})$. (3) The X-ray luminosity in the $2.0-10 \mathrm{keV}$ energy band is $\sim 10^{34} \mathrm{erg} \mathrm{s}^{-1}$ (4) The harder band (> $2 \mathrm{keV}$ ) emission dominates the spectrum. (5) The WD spin modulation is shown in the X-ray light curve [see figure [3]. (6) The spin modulation is larger in softer band and is thought to be due to absorption. The similarity to IPs suggests that V2491 Cyg has a magnetized WD. This agrees with a theoretical suggestion that the rebrightening event in optical decline is due to a sudden release of magnetic energy [Q].

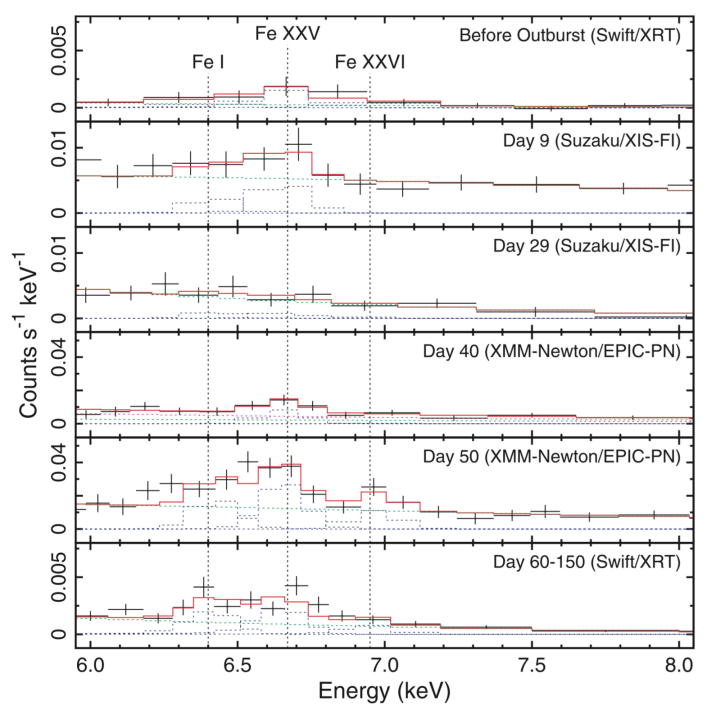

Figure 2: Narrow-band (6.0-8.0 keV) X-ray spectra of V2491 Cyg, including the Fe K complex, at six different epochs. The center energy of the three emission lines are indicated by dashed lines. Black crosses indicate the data, while the red solid histograms indicate the best-fit model. The model is decomposed into the power-law continuum (green dotted) and the three Gaussian line (blue dotted) components. The contamination by the nearby source in the XMM-Newton/EPIC-PN spectra on day 40 is shown as the dotted line in magenta. (Reproduction from fig.2 of [B] $]$ )

\subsection{V407 Cygni}

V407 Cygni is a symbiotic Mira star where a WD and a Mira-type asymptotic giant branch (AGB) companion make a binary system. The pulsation period of the Mira is $745 \pm 15$ days [[13]. The distance is estimated at from $1.9 \mathrm{kpc}$ [ए13] to $2.7 \mathrm{kpc}[\mathbb{1}]$ ] although hot bottom burning makes it difficult to measure. V407 Cyg has experienced two small-amplitude outbursts (peak visual brightness $\sim 13 \mathrm{mag}$ ) in 1936 and 1998. The 1998 event has been characterized as a classical symbiotic outburst. The other event was characterized as a nova but it seems doubt by present interpretation.

V407 Cyg caused the most recent outburst event on 2010 March 10 [ए0, 22] with a maximum brightness of $6.8 \mathrm{mag}$. The amplitude was much larger than the past events and, $t_{2}$ and $t_{3}$ were measured to be 5.9 and 24 days, respectively, making this event a fast nova. In the 2010 event, significant $\gamma$-ray emission was observed by Large Area Telescope (LAT) detector on board the Fermi observatory [U] between 2010 March 10 and 25. 

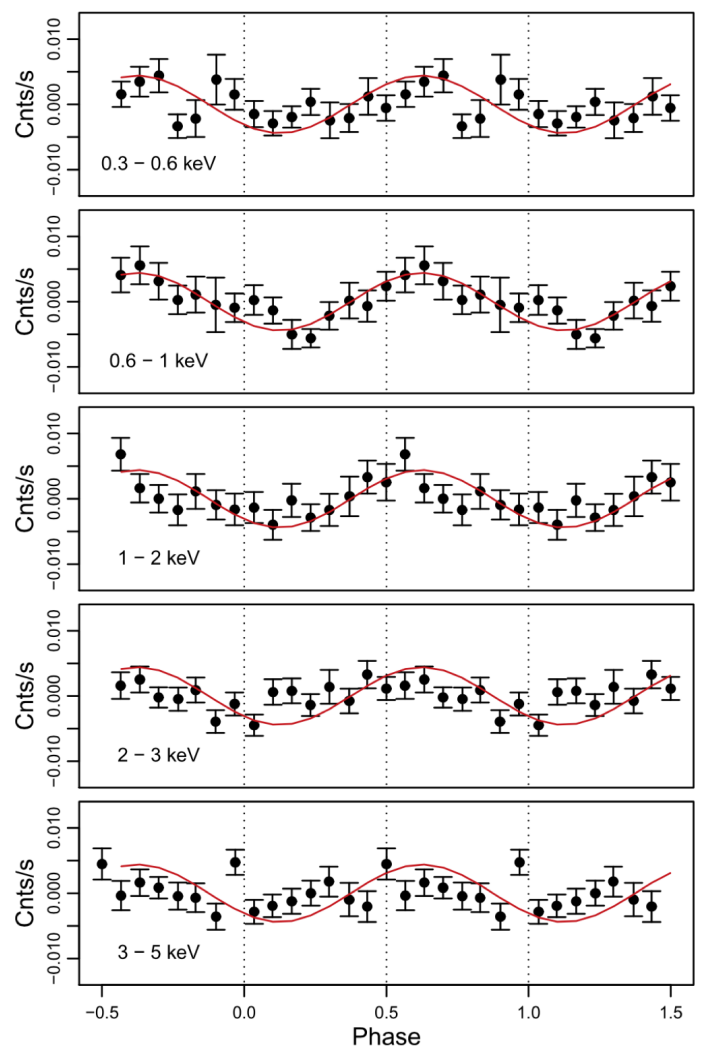

Figure 3: XIS-BI light curves of V2491 Cyg in different energy ranges folded with the period of 0.0266 days. The corresponding energy range is indicated in the bottom-left corner of each plot. (Reproduction from fig.6 of [42]])

Figure母 (reproduction from [23]) shows a X-ray spectrum of V407 Cyg observed by Suzaku at the peak of X-ray flux (day 30 of the outburst). A detailed spectroscopic analysis by the authors reveals hard and soft components. The first component dominates between 2 and $10 \mathrm{keV}$, and originates in the forward shock by interaction between the Mira wind and the ejecta. This component was discomposed into a collisional ionization equilibrium (CIE) emission with $T=2.3 \mathrm{keV}$ and a non-equilibrium ionization plasma (NEI) emission $T=4 \mathrm{keV}$ and $\tau=2.2 \times 10^{10} \mathrm{~cm}^{-3} \mathrm{~s}$. The presence of an NEI plasma is direct observational evidence of asymmetry in the environment. The second component peaks at energies below $1 \mathrm{keV}$ and, was identified to be supersoft X-ray emission from the white dwarf seen through the dense Mira wind.

The authors developed a simple model of the ejecta-environment interaction to show the rapid rise and subsequent decay of X-ray flux (see figure $[$ from [[23]). As a result, they explained the coincidental $\gamma$-ray decline/X-ray rise by the stalling of the shock front as the ejecta approaches the red giant photosphere.

\subsection{Symbiotic Stars}

CD -28 3719, Hen 3-1591, Hen 3-461 and EG And are SSs and their X-ray spectral types were classified into $\delta, \gamma, \delta$ and $\beta$, respectively [48, [5]]. 4 Dra is also a SS and its X-ray spectral type 


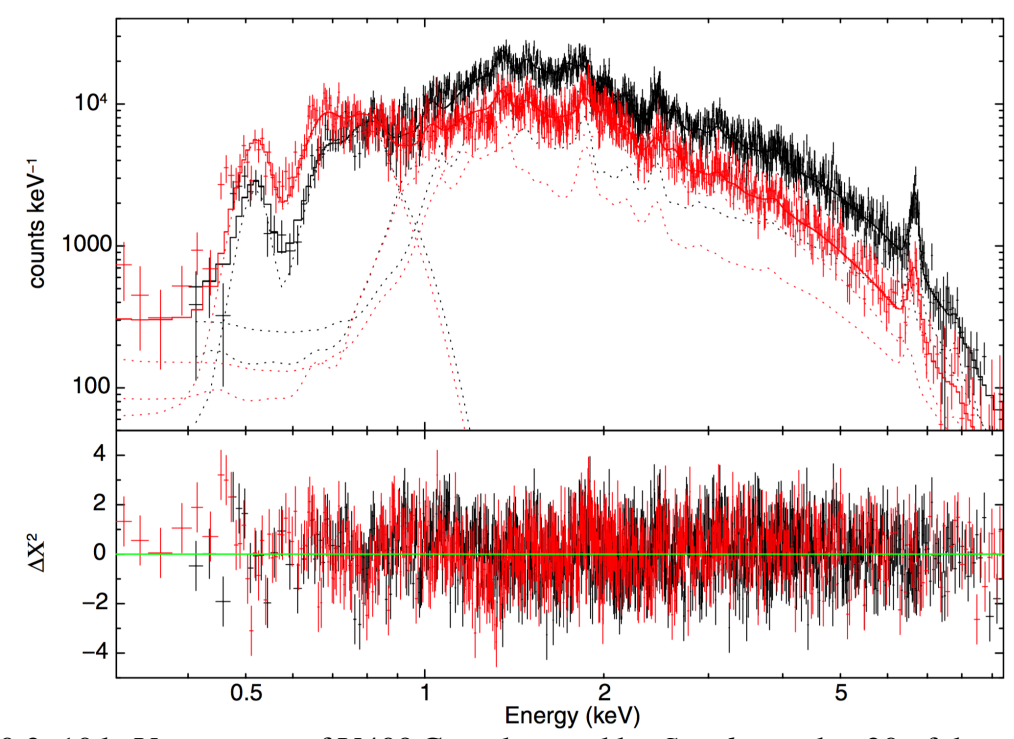

Figure 4: XIS 0.3-10 keV spectrum of V409 Cyg observed by Suzaku on day 30 of the outburst with best-fit model. This model has components of equilibrium, non-equilibrium ionization plasmas and blackbody. The lower panel shows the fit residuals in units of sigma. (Reproduction from fig.4 of [42])

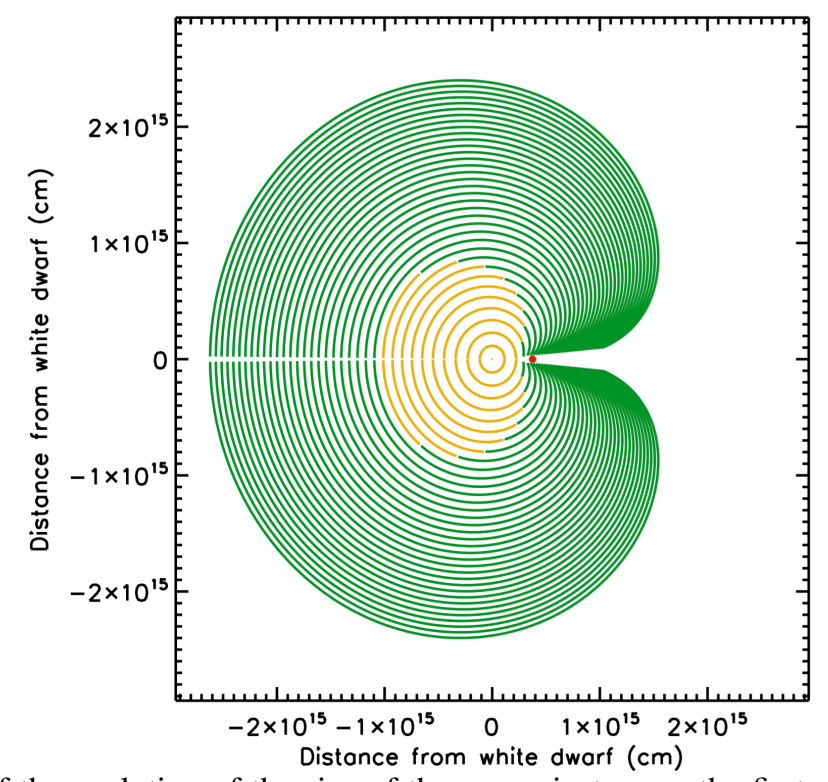

Figure 5: Example of the evolution of the size of the nova ejecta over the first $\sim 200$ days of the outburst in V409 Cyg, for an ejected mass of $5 \times 10^{-7} \mathrm{M}_{\odot}$. Each line corresponds to an increase of $100 \mathrm{hr}$ since the onset of the outburst. The red circle represents the giant, and its radius is to scale. Gold lines correspond to regions which are in free expansion, green lines to regions which are in the Sedov (energy conserving) phase. A binary separation of $25 \mathrm{AU}$ and a mass-loss rate from the giant of $10^{-7} \mathrm{M}_{\odot} \mathrm{yr}^{-1}$ are assumed. (Reproduction from fig.9 of [42]) 
was undefined. The five SSs were observed by Suzaku and their X-ray spectra are shown in figure 6 (reproduction from [24]).

Their X-ray spectra were successfully modeled by absorbed, optically thin thermal plasma emission of either a single- or multi- temperature. The maximum temperature of their plasma was estimated at 11, 6, 8, 3 and 7.3 keV for CD -28 3719, Hen 3-1591, Hen 3-461, EG And and 4 Dra, respectively. $\gamma$ and $\alpha$ type were rejected for the X-ray spectral types for the five SSs because of the high temperature $\left(T_{\max } \gtrsim 3 \mathrm{keV}\right)$. Assuming $\beta$ type, the relation between the shocked gas and the maximum temperature can be written by

$$
v_{\text {shock }}=(4 / 3) \times \sqrt{\frac{16 k T_{\max }}{3 \mu m_{\mathrm{H}}}} .
$$

The wind velocity is no more than $1000 \mathrm{~km} \mathrm{~s}^{-1}$ in general and, therefore, the $\beta$-type emission is difficult to explain the observed maximum temperature of all of the five SSs. On the other hand, assuming the $\delta$ type, the relation between the WD mass and the maximum temperature can be written by

$$
M_{\mathrm{WD}} \geq \frac{16 k T_{\max } R_{\mathrm{WD}}}{3 G \mu m_{\mathrm{H}}}
$$

With the WD mass of $0.4-0.6 M_{\odot}$, the all of observed maximum temperature can be explained by this mechanism. As a results, the X-ray spectral types of the five SSs were classified into $\delta$ in spite of the previous classification. Note that in Hen 3-461 a soft thermal component was also found below $2 \mathrm{keV}$ and it was classified into $\beta / \delta$ type.

\section{$3.4 \gamma$ Cas}

$\gamma$ Cas analogues are Be or Oe stars with intense X-ray emission [3]]. Their $L_{X} / L_{\text {bol }}$ ratio is a factor 10 higher than for normal OB stars $\left(L_{X} / L_{\mathrm{bol}} \sim 10^{-7}\right)$. Variability is found on many timescales from several second to several months. Their X-ray spectrum can be reproduced by a thermal plasma emission whose temperature is $T_{\max }>10 \mathrm{keV}$ or a combination of thermal plasma and power law emission. There are two model to produce the $\mathrm{X}$-ray emission. One is of magnetic star-circumstellar disk interactions [27] and the other is of accreting compact objects. About a dozen $\gamma$ Cas analogues were found up to the present.

Figure $\square$ shows X-ray spectrum of $\gamma$ Cas itself observed by Suzaku and INTEGRAL (reproduction from $[B]]$ ). The observed spectrum can be reproduced with a absorbed single-temperature plasma model of $T=15.37 \mathrm{keV}$ except for the fluorescent iron $\mathrm{K} \alpha$ line. With the model of the accreting compact object, if the compact object is a neutron star, the X-ray spectrum should have a component of power law. Therefore, a WD is more likely for the compact object.

Six dips were found in the $\gamma$ Cas X-ray light curve by Suzaku [24]). Four of them were observed from its start to end and, symmetric in shape in the light curve. The dip can be explained by partial covering absorption with different hydrogen column density for each dip $N_{\mathrm{H}}=$ $2-8 \times 10^{21} \mathrm{~cm}^{-2}$ and, on the other hand, covering fraction is $\sim 0.4$ or $\sim 0.7$ for all dips (see $\mathbb{8}$ ). Because of consistency between the covering fraction and duration of the dips, the authors concluded that there are two X-ray sources and multiple absorbers. Moreover, the absorbers are larger than the sources. The two of sources may not consistent with the magnetic star-disk dynamo theory 

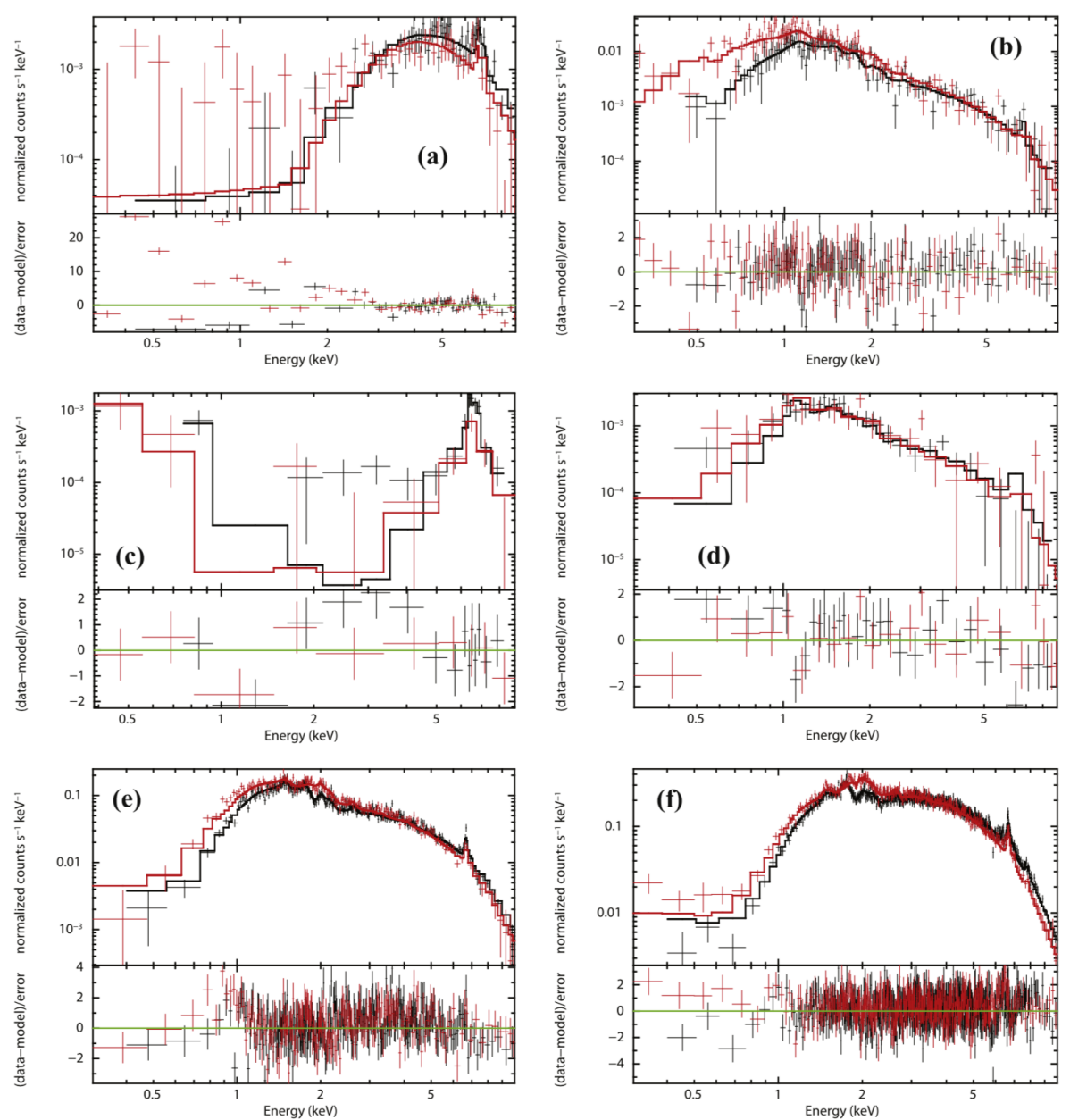

Figure 6: XIS spectra of (a) CD - 28 3719, (b) Hen 3-1591, (c) Hen 3-461, (d) EG And, (e) 4 Dra ObsID 4050, and (f) 4 Dra ObsID 4060. The solid lines show the best-fit models. Red shows XIS1 data; black shows XIS0+3 data. (Reproduction from fig.1 of [24])

[B2], in which X-rays originate from multiple magnetic loops on the Be stellar surface. With the magnetic star-circumstellar disk interaction model, the magnetic loops (i.e. X-ray source) should spread evenly over the Be star size and a single absorber should have a size of $\sim 0.4$ stellar radii $\left(4 R_{\odot}\right)$. A projection velocity of the absorber should be up to $\sim 5000 \mathrm{~km} \mathrm{~s}^{-1}$, which is unrealistic for the wind or disk of the Be star. On the other hand, the small numbers of discrete source fit with the accreting compact star model. The thermal X-ray spectrum of $T=15 \mathrm{keV}$ is similar to WD binaries and, therefore, a accreting WD is more likely as mentioned above.

\section{Summary}

Suzaku observed dozens of WD binaries until it completed its scientific operation in June 2015. In this paper, four topics are picked up and introduced in brief.

In a nova outburst of V2491 Cyg, a very hard non-thermal component with $\Gamma=0.1 \pm 0.2$ was observed and it was observationally verified that novae are a particle-accelerate cite. X-ray origin 


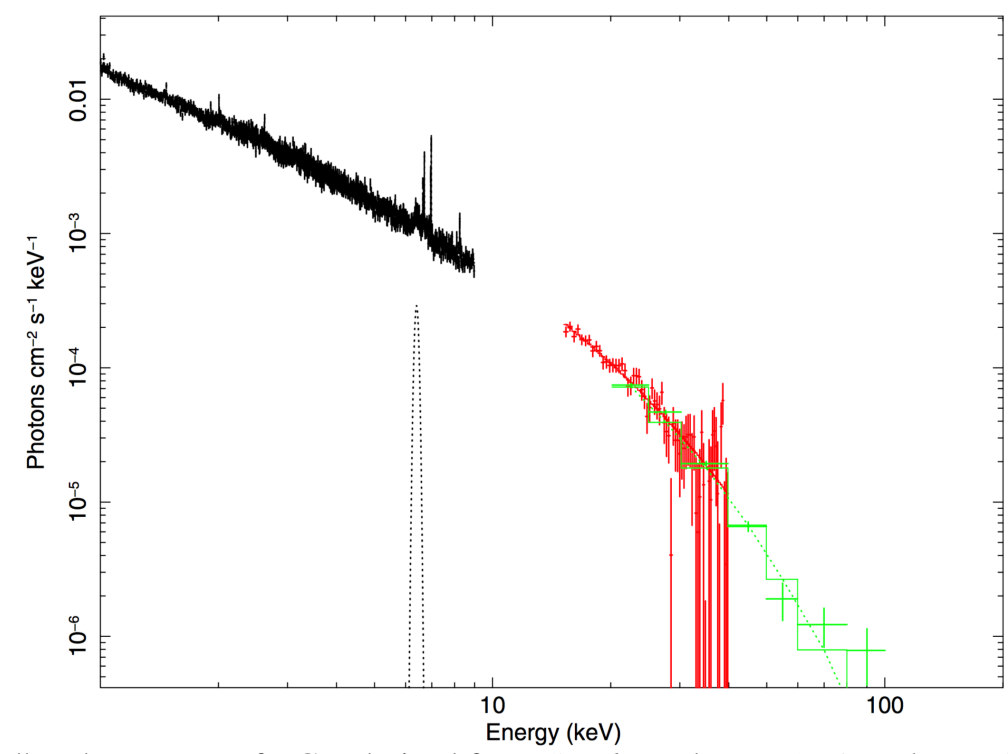

Figure 7: Broadband spectrum of $\gamma$ Cas derived from Suzaku and INTEGRAL. The model consists of emission from thermal plasma with temperature of $15.3 \mathrm{keV}$ a Gaussian line feature at $6.4 \mathrm{keV}$, and a single absorption component. Clearly, the model represents the data extremely well. (Reproduction from fig.5 of [B] $]$ )

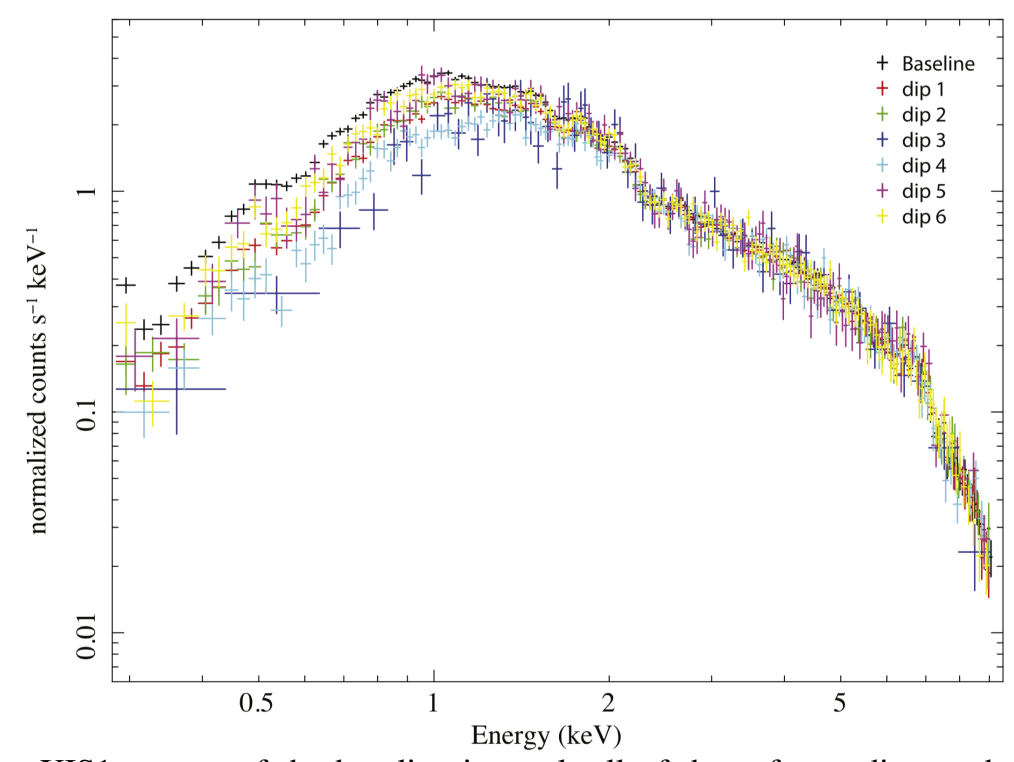

Figure 8: $\gamma$ Cas XIS1 spectra of the baseline interval, all of the softness dips, and the stable emission normalized with the best-fit normalization values of the hard variable component. (Reproduction from fig. 6 of [24]) 
changed from internal shocks to mass accretion at the peak in X-ray flux (40 days after the nova start) by rekindled accretion. In fact, several features of the X-ray emission are similar to those of IP, which support a theoretical suggestion that the optical rebrightening in the nova declining is due to magnetic activity.

At the X-ray peak (day 30) of the nova outburst of a symbiotic Mira star V407 Cyg, NEI plasma emission was found in the X-ray spectrum in addition to CIE plasma emission. The presence of the NEI plasma shows asymmetry in the environment encountered by the nova ejecta. The coincidental GeV $\gamma$-ray decline/X-ray rise can be explained by the stalling of the shock front as the ejecta approaches the red giant photosphere.

X-ray spectra of five symbiotic stars (CD-28 3719, Hen 3-1591, Hen 3-461, EG And and 4 Dra) were acquired by Suzaku. All of the five SSs have hot thermal plasma of $T_{\max } \gtrsim 3 \mathrm{keV}$. The temperature can not be reproduced by the quasi-stable nuclear burning on the WD surface or the collision of the wind. As a result, the X-ray spectrum of the five SSs were classified into the release of the gravitational potential in the boundary layer (i.e. $\delta$ type). Note that Hen 3-461 was classified into $\beta / \delta$ type because of soft thermal component below $2 \mathrm{keV}$.

$\mathrm{X}$-ray spectrum of $\gamma$ Cas up to $100 \mathrm{keV}$ can be reproduced by a single-temperature thermal plasma model of $15 \mathrm{keV}$. If $\gamma$ Cas is a neutron star binary, its X-ray spectrum has a component of power law. Therefore, neutron star binary is not realistic for identity of $\gamma$ Cas. Dips of order of $10^{4} \mathrm{~s}$ in X-ray light curve can be explained by partial covering absorption. The size of absorbers should be larger than X-ray sources because of the covering fraction from the fitting and dip duration. Assuming the magnetic star-circumstellar disk interaction model, the absorbers should be $\sim 0.4$ stellar radii $\left(4 R_{\odot}\right)$ in size and their velocity is about $5000 \mathrm{~km} \mathrm{~s}^{-1}$, which is nonrealistic. As a result, a WD binary is more likely for $\gamma$ Cas identity.

\section{References}

[1] Abdo A. A., et al., 2010, Sci, 329, 817

[2] Aizu, K. 1973, Progress of Theoretical Physics, 49, 1184

[3] Belczyński K., Mikołajewska J., Munari U., Ivison R. J., Friedjung M., 2000, A\&AS, 146, 407

[4] Cropper, M. 1990, SSRv, 54, 195

[5] della Valle M., Livio M., 1995, ApJ, 452, 704

[6] Gehrz R. D., Truran J. W., Williams R. E., Starrfield S., 1998, PASP, 110, 3

[7] George, I. M., \& Fabian, A. C. 1991, MNRAS, 249, 352

[8] Hōshi, R. 1973, Progress of Theoretical Physics, 49, 776

[9] Hachisu I., Kato M., 2009, ApJ, 694, L103

[10] Hirosawa K., 2010, CBET, 2199, 1

[11] Kokubun, M., Makishima, K., Takahashi, T., et al. 2007, PASJ, 59, 53

[12] Koyama, K., Tsunemi, H., Dotani, T., et al. 2007, PASJ, 59, 23

[13] Kolotilov E. A., Munari U., Popova A. A., Tatarnikov A. M., Shenavrin V. I., Yudin B. F., 1998, AstL, 24, 451 
[14] Masetti, N., Orlandini, M., Palazzi, E., Amati, L., \& Frontera, F. 2006, A\&A, 453, 295

[15] Luna G. J. M., Sokoloski J. L., Mukai K., Nelson T., 2013, A\&A, 559, A6

[16] Mattana, F., Götz, D., Falanga, M., et al. 2006, A\&A, 460, L1

[17] Mitsuda, K., Bautz, M., Inoue, H., et al. 2007, PASJ, 59, 1

[18] Muerset U., Wolff B., Jordan S., 1997, A\&A, 319, 201

[19] Munari U., Margoni R., Stagni R., 1990, MNRAS, 242, 653

[20] Nakajima, H., Yamaguchi, H., Matsumoto, H., et al. 2008, PASJ, 60, S1

[21] Nakano S., et al., 2008, IAUC, 8934, 1

[22] Nakano S., 2010, CBET, 2199, 2

[23] Nelson T., Donato D., Mukai K., Sokoloski J., Chomiuk L., 2012, ApJ, 748, 43

[24] Nuñez N. E., Nelson T., Mukai K., Sokoloski J. L., Luna G. J. M., 2016, ApJ, 824, 23

[25] Osaki Y., 1974, PASJ, 26, 429

[26] Patterson, J. 1994, PASP, 106, 209

[27] Robinson R. D., Smith M. A., Henry G. W., 2002, ApJ, 575, 435

[28] Samus N. N., 2008, IAUC, 8934, 2

[29] Serlemitsos, P. J., Soong, Y., Chan, K.-W., et al. 2007, PASJ, 59, 9

[30] Shrader C. R., Hamaguchi K., Sturner S. J., Oskinova L. M., Almeyda T., Petre R., 2015, ApJ, 799, 84

[31] Smith M. A., et al., 2012, A\&A, 540, A53

[32] Smith M. A., Lopes de Oliveira R., Motch C., 2016, AdSpR, 58, 782

[33] Takahashi, T., Abe, K., Endo, M., et al. 2007, PASJ, 59, 35

[34] Takahashi, T., Mitsuda, K., Kelley, R., et al. 2010, SPIE proc, 7732, 77320Z

[35] Takei D., Tsujimoto M., Kitamoto S., Ness J.-U., Drake J. J., Takahashi H., Mukai K., 2009, ApJ, 697, L54

[36] Takei D., Ness J.-U., Tsujimoto M., Kitamoto S., Drake J. J., Osborne J. P., Takahashi H., Kinugasa K., 2011, PASJ, 63, S729

[37] Tomov T., Mikolajewski M., Brozek T., Ragan E., Swierczynski E., Wychudzki P., Galan C., 2008, ATel, 1485,

[38] Uchiyama, H., Ozawa, M., Matsumoto, H., et al. 2009, PASJ, 61, S9

[39] Wada Q., Tsujimoto M., Ebisawa K., Hayashi T., 2017, PASJ, 69, 10

[40] Warner B., 1995, CAS, 28,

[41] Yuasa T., Nakazawa K., Makishima K., Saitou K., Ishida M., Ebisawa K., Mori H., Yamada S., 2010, A\&A, 520, A25

[42] Zemko P., Mukai K., Orio M., 2015, ApJ, 807, 61 